\title{
$X X V$. On the conduction of heat in a spherical mass of air confined by walls at a constant temperature
}

\section{Lord Rayleigh F.R.S.}

To cite this article: Lord Rayleigh F.R.S. (1899) XXV. On the conduction of heat in a spherical mass of air confined by walls at a constant temperature, Philosophical Magazine Series 5 , 47:286, 314-325, DOI: 10.1080/14786449908621264

To link to this article: http://dx.doi.org/10.1080/14786449908621264

曲 Published online: 08 May 2009.

Submit your article to this journal $₫$

Џ Article views: 6

Q View related articles $\square$

Citing articles: 1 View citing articles 4 


\section{Lord Rayleigh on Conduction of Heat in a Spherical}

thermal capacity of unit volume. This thermal capacity is to be taken with volume constant, and it will be less than the thermal capacity with pressure constant in the ratio of $\gamma: 1$. Accordingly $v / \gamma$ in (6) represents the latter thermal capacity, of which the experimental value is $\cdot 00128 \times \cdot 239$, the first factor representing the density of air referred to water. Thus, if we take the calorimetric conductivity at 000056 . we have in c.G.s. measure

and thence

$$
\nu=\cdot 258, \quad \nu / \gamma=\cdot 183 ;
$$

$$
t=\cdot 102 a^{2} \text {. }
$$

In the present apparatus $a$, determined by the contents, is 16.4 centim., whence

$$
t=27 \cdot 4 \text { seconds. }
$$

The agreement of the observed and calculated values is quite as close as could have been expected, and confirms the view that the transfer of heat is due to conduction, and that the part played by radiation is insensible. From a comparison of the experimental and calculated curves, however, it seems probable that the effect of gravity was not wholly eliminated, and that the later stages of the phenomenon, at any rate, may still have been a little influenced by a downward movement of the central parts.

XXV. On the Conduction of Heat in a Spherical Mass of Air confined by Walls at a Constant Temperature. By Lord RAYLEIGH, $\boldsymbol{F} . R . S . *$

T $\mathrm{T}$ is proposed to investigate the subsidence to thermal 1 equilibriam of a gas slightly disturbed therefrom and included in a solid vessel whose walls retain a constant temperature. The problem differs from those considered by Fourier in consequence of the mobility of the gas, which may give rise to two kinds of complication. In the first place gravity; taking advantage of the different densities prevailing in various parts, tends to produce circulation. In many cases the subsidence to equilibrium must be greatly modified thereby. But this effect diminishes with the amount of the temperature disturbance, and for infinitesimal disturbances the influence of gravity disappears. On the other hand, the second complication remains, even though we limit ourselves to infinitesimal disturbances. When one part of the gas expands in consequence of reception of heat by

* Communicated by the Aulhor. 
radiation or conduction, it compresses the remaining parts, and these in their turn become heated in accordance with the laws of gases. To take account of this effect a special investigation is necessary.

But although the fixity of the boundary does not suffice to prevent local expansions and contractions and consequent motions of the gas, we may nevertheless neglect the inertia of these motions since they are very slow in comparison with the free oscillations of the mass regarded as a resonator. Accordingly the pressure, although variable with time, may be treated as uniform at any one moment throughout the mass.

In the usual notation *, if $s$ be the condensation and $\theta$ the excess of temperature, the pressure $p$ is given by

$$
p=k \rho(1+s+\alpha \theta) \text {. }
$$

The effect of a small sudden condensation $s$ is to produce an elevation of temperature, which may be denoted by $\beta s$. Let $d Q$ be the quantity of heat entering the element of volume in the time $d t$, measured by the rise of temperature which it would produce, if there were no " condensation." Then

$$
\frac{d \theta}{d t}=\beta \frac{d s}{d t}+\frac{d \mathrm{Q}}{d t} ; . . . \quad . \quad . \quad .
$$

and, if the passage of $d Q$ be the result of radiation and conduction, we have

$$
\frac{d \mathrm{Q}}{d t}=\nu \nabla^{2} \theta-q \theta . \quad \text {. . . . . }
$$

In (3) $v$ represents the "thermometric conductivity" found by dividing the conductivity by the thermal capacity of the gas (per unit volume), at constant volume. Its value for air at $0^{\circ}$ and atmospheric pressure may be taken to be $\cdot \mathbf{2 6}$ $\mathrm{cm}^{2}$./sec. Also $q$ represents the radiation, supposed to depend only upon the excess of temperature of the gas over that of the enclosure.

If $d \mathrm{Q}=0, \theta=\beta s$, and in (1)

so that

$$
p=k \rho\{1+(1+\alpha \beta) s\} ;
$$

$$
1+\alpha \beta=\gamma,
$$

where $\boldsymbol{\gamma}$ is the well-known ratio of specific heats, whose value for air, and several other gases is very nearly $1 \cdot 41$.

In general from (2) and (3)

$$
\frac{d \theta}{d t}=\beta \frac{d s}{d t}+\nu \nabla^{2} \theta-q \theta \text {. . . . . . }
$$

* 'Theory of Sound,' $\$ 247$. 
316 Lord Rayleigh on Conduction of Heat in a Spherical

In order to find the normal modes into which the most general subsidence may be analysed, we are to assume that 8 and $\theta$ are functions of the time solely through the factor $e^{-h t}$. Since $p$ is uniform, $s+\alpha \theta$ must by (1) be of the form $\mathrm{H} e^{-h t}$, where $\mathrm{H}$ is some constant; so that if for brevity the factor $e^{-h t}$ be dropped,

while from (5)

$$
s+a \theta=\mathrm{H} ; . . .2 . . .
$$

$$
\nu \nabla^{2} \theta+(h-q) \theta=h \beta s . . . \quad . \quad .
$$

Fliminating $s$ between (5) and (7), we get

where

$$
\nabla^{2} \theta+m^{2}(\theta-\mathrm{C})=0 \text {, . . }
$$

$$
m^{2}=\frac{h \gamma-q}{\nu}, \quad \mathrm{C}=\frac{h \beta \mathrm{H}}{h \gamma-q} . \quad . \quad . \quad .
$$

These equations are applicable in the general case, but when radiation and conduction are both operative the equation by which $m$ is determined becomes rather complicated. If there be no conduction, $\nu=0$. The solution is then very simple, and may be worth a moment's attention.

Equations (6) and (7) give

$$
\theta=\frac{h \beta \mathrm{H}}{h \gamma-q}, \quad s=\frac{(h-q) \mathrm{H}}{h \gamma-q} . . .
$$

Now the mean value of $s$ throughout the mass, which does not change with the time, must be zero; so that from (10) we obtain the alternatives

$$
\text { (i.) } h=q, \quad \text { (ii.) } \mathrm{H}=0 \text {. }
$$

Corresponding to (i.) we have with restoration of the timefactor

$$
\theta=(\mathrm{H} / \alpha) e^{-q t}, \quad s=0 . \quad \text {. . . . }
$$

In this solution the temperature is uniform and the condensation zero throughout the mass. By means of it any initial mean temperature may be provided for, so that in the remaining solutions the mean temperature may be considered to be zero.

In the second alternative $\mathrm{H}=0$, so that $s=-\alpha \theta$. Using this in (7) with $v$ evanescent, we get

$$
\left(h_{\gamma}-q\right) \theta=0 \text {. . . . . . . }
$$

The second solution is accordingly

$$
\theta=\phi(x, y, z) e^{-q t / \gamma}, \quad s=-\alpha \phi(x, y, z) e^{-q t / \gamma}, .
$$

where $\phi$ denotes a function arbitrary throughout the mass, except for the restriction that its mean value must be zero. 
Mass of Air confined by Walls at Constant Temperature. 317

Thus if $\Theta$ denote the initial value of $\theta$ as a function of $x, y, z$, and $\Theta_{0}$ its mean value, the complete solution may be written

giving

$$
\left.\begin{array}{l}
\theta=\Theta_{0} e^{-q t}+\left(\Theta-\Theta_{0}\right) e^{-q t_{i} / \gamma} \\
s=-\alpha\left(\Theta-\Theta_{0}\right) e^{-q t / \gamma}
\end{array}\right\}, . . .
$$

$$
s+\alpha \theta=\alpha \Theta_{u} e^{-q t} \text {. . . . . . }
$$

It is on (15) that the variable part of the pressure depends.

When the conductivity $v$ is finite, the solutions are less simple and involve the form of the vessel in which the gas is contained. As a first example we may take the case of gas bounded by two parallel planes perpendicular to $x$, the temperature and condensation being even functions of $x$ measured from the mid-plane. In this case $\nabla^{2}=d^{2} / d x^{2}$, and we get

By (9), (17)

$$
\begin{array}{r}
\theta=\mathrm{C}+\mathrm{A} \cos m x, \quad-s / \alpha=\mathrm{D}+\mathrm{A} \cos m x, . \\
s+\alpha \theta=\alpha \mathrm{C}-\alpha \mathrm{D}=\mathrm{H} . . .
\end{array}
$$

$$
\mathrm{C}=\frac{h \beta \mathrm{H}}{l_{\gamma}-q}, \quad \mathrm{D}=\frac{(q-h) \mathrm{H}}{\alpha(h \gamma-q)} . \quad . \quad .
$$

There remain two conditions to be satisfied. The first is simply that $\theta=0$ when $x= \pm a, 2 a$ being the distance between the walls. This gives

$$
\mathrm{C}+\mathrm{A} \cos m a=0 \text {. }
$$

The remaining condition is given by the consideration that the mean value of $s$, proportional to $\int s d x$, must vanish. Accordingly

$$
m a \cdot \mathrm{D}+\sin m a \cdot \mathrm{A}=0 .
$$

From $(18),(19),(20)$ we have as the equation for the admissible values of $m$,

$$
\frac{\tan m a}{m a}=\frac{a \beta q-\nu m m^{2}}{\alpha \beta\left(\hat{q}+\nu m^{2}\right)}, \quad . \quad . \quad . \quad .
$$

reducing for the case of evanescent $q$ to

$$
\frac{\tan m a}{m a}=-\frac{1}{a \beta} . \text {. . . . . }
$$

The general solntion may be expressed in the series

$$
\left.\begin{array}{l}
\theta=\mathrm{A}_{1} e^{-h_{1} t} \theta_{1}+\mathrm{A}_{2} e^{-h_{2} t} \theta_{2}+\ldots \\
s=\mathrm{A}_{1} e^{-h_{1} t} s_{1}+\mathrm{A}_{2} e^{-h_{2} t} s_{2}+\ldots
\end{array}\right\}, . . .
$$

Phil. Mag. S. 5. Vol, 47. No 286. March 1899. 


\section{Lord Rayleigh on Conduction of Heat in a Spherical}

where $h_{1}, h_{2}, \ldots$ are the values of $h$ corresponding according to (9) with the various values of $m$, and $\theta_{1}, \theta_{2} \ldots$ are of the form

$$
\left.\begin{array}{l}
\theta_{1}=\cos m_{1} x-\cos m_{1} a \\
s_{1}=-\alpha\left(\cos m_{1} x-\sin m_{1} a / m_{1} a\right)
\end{array}\right\} \text {. . . . }
$$

It only remains to determine the arbitrary constants $A_{1}$, $A_{2}, \ldots$ to suit prescribed initial conditions. We will limit ourselves to the simpler case of $q=0$, so that the values of $m$ are given by (22). With use of this relation and putting for brevity $a=1$, we find from (24)

so that

$$
\begin{aligned}
& \int_{0}^{1} \theta_{1} \theta_{2} d x=\frac{\alpha \beta+1}{\alpha \beta} \cos m_{1} \cos m_{2}, \\
& \int_{0}^{1} s_{1} s_{2} d x=-\frac{\alpha \beta+1}{\beta^{2}} \cos m_{1} \cos m_{2} ;
\end{aligned}
$$

$$
\int_{0}^{1} \theta_{1} \theta_{2} d x+\beta / a \cdot \int_{0}^{1} s_{1} s_{2} d x=0, \ldots . . .
$$

$\theta_{1}, \theta_{2}$ being any (different) functions of the form (24). Also

$$
\int_{0}^{1} \theta_{1}^{2} d x+\beta / \alpha \cdot \int_{0}^{1} s_{1}^{2} d x=\frac{1+\alpha \beta}{2}\left\{1+\frac{\cos ^{2} m_{1}}{\alpha \beta}\right\} .
$$

There is now no difficulty in finding $A_{1}, A_{2}, \ldots$ to suit arbitrary initial values of $\theta$ and its associated $s, i, e$. so that

$$
\left.\begin{array}{l}
\Theta=A_{1} \theta_{1}+A_{2} \theta_{2}+\ldots \\
\mathbf{S}=A_{1} s_{1}+A_{2} s_{2}+\ldots
\end{array}\right\} . . . .
$$

Thus to determine $A_{1}$,

$$
\begin{aligned}
\int_{0}^{1}\left(\Theta \theta_{1}+\beta / \alpha\right. & \left.. \mathrm{S} s_{1}\right) d x=A_{1} \int_{0}^{1}\left(\theta_{1}{ }^{2}+\beta / \alpha . s_{1}{ }^{2}\right) d x \\
& +A_{2} \int_{0}^{1}\left(\theta_{1} \theta_{2}+\beta / \alpha . s_{1} s_{2}\right) d x+\ldots \ldots
\end{aligned}
$$

in which the coefficients of $A_{2}, A_{3} \ldots$ vanish by (25); so that by (26)

$$
A_{1}\left\{1+\frac{\cos ^{2} m_{1}}{\alpha \beta}\right\}=\frac{2}{1+\alpha \beta} \int_{0}^{1}\left(\Theta \theta_{1}+\beta / \alpha . S s_{1}\right) d x .
$$

An important particular case is that in which $\Theta$ is constant, and accordingly $S=0$. Since

$$
\int_{0}^{1} \theta_{1} d x=\frac{\sin m_{1}}{m_{1}}-\cos m_{1}=-\frac{1+\alpha \beta}{\alpha \beta} \cos m_{1},
$$


Mass of Air confined by Walls at Constant Temperature. 319 we have

$$
A_{1}=-\frac{2 \Theta \cos m_{1}}{\alpha \beta+\cos ^{2} m_{1}} \text {. . . . . . }
$$

For the pressure we have

$$
\begin{aligned}
\theta+s / \alpha & =A_{1} e^{-k_{1} t}\left(-\cos m_{1}+\frac{\sin m_{1}}{m_{1}}\right)+\ldots \\
& =-\frac{\alpha \beta+1}{\alpha \beta} \cos m_{1} \cdot A_{1} e^{-k_{1} t}+\ldots \ldots,
\end{aligned}
$$

or in the particular case of (29),

$$
\theta+s / \alpha=2 \Theta \frac{1+\alpha \beta}{\alpha \beta} \frac{\cos ^{2} m_{1} e^{-\lambda_{1} t}}{\alpha \beta+\cos ^{2} m_{1}}+\ldots .
$$

If $\beta=0$, we fall back upon a problem of the Fourier type. By (22) in that case

and

$$
\begin{gathered}
m a=\frac{1}{2} \pi(1,3,5, \ldots) \\
\cos ^{2} m a=\alpha^{2} \beta^{2} / m^{2} a^{2},
\end{gathered}
$$

so that (30) becomes

or initially

$$
2 \Theta\left(\frac{e^{-\lambda_{1} t}}{m_{1}^{2} a^{2}}+\frac{e^{-\lambda_{2} t}}{m_{2}^{2} a^{2}}+\ldots\right), \quad . \quad . .
$$

$$
\frac{8 \Theta}{\pi^{2}}\left(\frac{1}{1^{2}}+\frac{1}{3^{2}}+\frac{1}{\overline{5}^{2}}+\ldots\right), \text { i.e. } \Theta \text {. }
$$

The values of $h$ are given by

$$
h=\frac{v \pi^{2}}{4 \gamma a^{2}}\left(1^{2}, 3^{2}, 5^{2}, \ldots\right) \text {. . . . . }
$$

We will now pass on to the more important practical case of a spherical envelope of radius $a$. The equation (8) for $(\theta-C)$ is identical with that which determines the vibrations of air ${ }^{*}$ in a spherical case, and the solution may be expanded in Laplace's series. The typical term is

$$
\theta-\mathrm{C}=(m r)^{-\frac{1}{2}} J_{n+\frac{1}{3}}(m r) \cdot \mathrm{Y}_{n}, \cdot . \cdot
$$

$Y_{n}$ being the surface spherical harmonic of order $n$ where $n=0,1,2,3 \ldots$, and $J$ the symbol of Bessel's functions. In virtue of (6) we may as before equate $-s / \alpha-D$, where $D$ is another constant, to the right-hand member of (33). The two conditions yet to be satisfied are that $\theta=0$ when $r=a$, and that the mean value of $s$ throughout the sphere shall vanish.

* 'Theory of Sound,' vol. II. ch. xvii. 
320 Lord Rayleigh on Conduction of Heat in a Spherical

When the value of $n$ is greater than zero, the first of these. conditions gives $\mathrm{C}=0$ and the second $\mathrm{D}=0$; so that

$$
\theta=-s / \alpha=(m r)^{-\frac{1}{2}} \mathrm{~J}_{n+\frac{1}{2}}(m r) . \mathrm{Y}_{n}, . .
$$

and $s+\alpha \theta=0$. Accordingly these terms contribute nothing to the pressure. It is further required that

$$
\mathrm{J}_{n+\frac{1}{2}}(m a)=0
$$

by which the admissible values of $m$ are determined. The roots of (35) are discussed in 'Theory of Sound,' $\$ 206 \ldots$. ; kut it is not necessary to go further into the matter here, as interest centres rather upon the case $n=0$.

If we assume symmetry with respect to the centre of the sphere, we may replace $\nabla^{2}$ in (8) by $\frac{1}{r} \frac{d^{9}}{d r^{2}} r$, thus obtaining

$$
\frac{d^{2} r(\theta-\mathrm{C})}{d r^{2}}+m^{2} r(\theta-\mathrm{C})=0, \quad . \quad . \quad .
$$

of which the general solution is

$$
\theta=\mathrm{C}+\mathrm{A} \frac{\cos m r}{m r}+\mathrm{B} \frac{\sin m r}{m r} .
$$

But for the present purpose the term $r^{-1} \cos m r$ is excluded, so that we may write

giving

$$
\theta=\mathrm{C}+\mathrm{B} \frac{\sin m r}{m r}, \quad-s / \alpha=\mathrm{D}+\mathrm{B} \frac{\sin m r}{m r}, \quad .
$$

$$
s+\alpha \theta=\alpha(\mathrm{C}-\mathrm{D})=\mathrm{H} . \quad . \quad .
$$

The first special condition gives

$$
m a \mathrm{C}+\mathrm{B} \sin m a=0 \text {. . . . . . . }
$$

The second, that the mean value of $s$ shall vanish, gives on integration

$$
\frac{1}{3} m^{3} a^{3} \mathrm{D}+\mathrm{B}(\sin m a-m a \cos m a)=0 .
$$

Equations (18), derived from (9) and (37 bis), giving $\mathrm{C}$ and $\mathrm{D}$ in terms of $\mathrm{H}$, hold good as before. Thus

$$
\underset{\mathrm{C}}{\mathrm{D}}=\frac{q-h}{h a \beta}=\frac{\alpha \beta q-\nu m^{2}}{\alpha \beta\left(q+\nu m^{2}\right)} . . . \quad . \quad .
$$

Fiquating this ratio to that derived from (38), (39), we find

$$
\frac{3}{m^{2} a^{2}} \frac{m a \cos m a-\sin m a}{\sin m a}=\frac{\nu m^{2}-\alpha \beta q}{\alpha \beta\left(\nu m^{2}+q\right)} . .
$$


Mass of Air confined by Walls at Constant Temperature. 321 This is the equation from which $m$ is to be found, after which $h$ is given by (9).

In the further discussion we will limit ourselves to the case of $q=0$, when (41) reduces to

$$
m^{2}=3 \alpha \beta(m \cot m-1), \text {. . . }
$$

in which $a$ has been put equal to unity. Here by (40)

$$
\mathrm{D}=-\mathrm{C} / \alpha \beta \text {. }
$$

Thus we may set as in (23),

$$
\left.\begin{array}{l}
\theta=\mathrm{B}_{1} e^{-h_{1} t} \theta_{1}+\mathrm{B}_{2} e^{-h_{2} t} \theta_{2}+\ldots \ldots \\
s=\mathrm{B}_{1} e^{-h_{1} t} s_{1}+\mathrm{B}_{9} e^{-h_{2} t_{2}}+\ldots \ldots
\end{array}\right\},
$$

in which

$$
\theta_{1}=\frac{\sin m_{1} r}{m_{1} r}-\frac{\sin m_{1} a}{m_{1} a}, \quad s_{1}=-\alpha \frac{\sin m_{1} r}{m_{1} r}-\frac{1}{\beta} \frac{\sin m_{1} a}{m_{1} a}
$$

and by (9) $h_{1}=v m_{1}^{2} / \gamma$. Also

$$
s_{1} / \alpha+\theta_{1}=-\frac{1+\alpha \beta}{\alpha \beta} \frac{\sin m_{1} a}{m_{1} a} . . . . .
$$

The process for determining $B_{1}, B_{2}, \ldots$ follows the same lines as before. By direct integration from (44) we find

$$
\begin{aligned}
& \frac{2 m_{1} m_{2}}{1+\alpha \beta} \int_{0}^{1}\left(\theta_{1} \theta_{2}+\beta / \alpha \cdot s_{1} s_{2}\right) r^{2} d r \\
& \quad=\frac{\sin \left(m_{1}-m_{2}\right)}{m_{1}-m_{2}}-\frac{\sin \left(m_{1}+m_{2}\right)}{m_{1}+m_{2}}+\frac{2 \sin m_{1} \sin m_{2}}{3 \alpha \beta},
\end{aligned}
$$

$a$ being put equal to unity. By means of equation (42) satisfied by $m_{1}$ and $m_{2}$ we may show that the quantity on the right in the above equation vanishes. For the sum of the first two fractions is

$$
\frac{2 m_{2} \sin m_{1} \cos m_{2}-2 m_{1} \sin m_{2} \cos m_{1}}{m_{1}^{2}-m_{2}^{2}},
$$

of which the denominator by (42) is equal to

$$
3 \alpha \beta\left(m_{1} \cot m_{1}-m_{2} \cot m_{2}\right) \text {. }
$$

$$
\text { Accordingly } \int_{0}^{1}\left(\theta_{1} \theta_{2}+\beta / \alpha \cdot s_{1} s_{2}\right) r^{2} d r=0 . \quad \text {. . }
$$


322 Lord Rayleigh on Conduction of Heat in a Spherical

Also

$\frac{2 m_{1}^{2}}{1+\alpha \beta} \int_{0}^{1}\left(\theta_{1}^{2}+\beta / \alpha \cdot s_{1}^{2}\right) r^{2} d r=1-\frac{\sin 2 m_{1}}{2 m_{1}}+\frac{2 \sin ^{2} m_{1}}{3 \alpha \beta}$.

To determine the arbitrary constants $B_{1} \ldots$ from the given initial values of $\theta$ and $s$, say $\Theta$ and $\mathrm{S}$, we proceed as usual. We limit ourselves to the term of zero order in spherical harmonics, $i$.e. to the supposition that $\theta, s$ are functions of $r$ only. The terms of higher order in spherical harmonics, if present, are treated more easily, exactly as in the ordinary theory of the conduction of heat. By (43)

and thus

$$
\left.\begin{array}{l}
\Theta=\mathrm{B}_{1} \theta_{1}+\mathrm{B}_{2} \theta_{2}+\ldots . \\
\mathrm{S}=\mathrm{B}_{1} s_{1}+\mathrm{B}_{2} s_{2}+\ldots .
\end{array}\right\} ; . \text {. . . }
$$

$$
\begin{gathered}
\int_{0}^{1}\left(\Theta \theta_{1}+\beta / \alpha \cdot \mathrm{S} s_{1}\right) r^{2} d r=\mathrm{B}_{1} \int_{0}^{1}\left(\theta_{1}^{2}+\beta / \alpha \cdot s_{1}^{2}\right) r^{2} d r \\
+\mathrm{B}_{2} \int_{0}^{1}\left(\theta_{1} \theta_{2}+\beta / \alpha \cdot s_{1} s_{2}\right) r^{2} d r+\ldots,
\end{gathered}
$$

in which the coefficients of $B_{2}, B_{3}, \ldots$ vanish by (46). The coefficient of $B_{1}$ is given by (47). Thus

$\mathrm{B}_{1}\left\{1-\frac{\sin 2 m_{1}}{2 m_{1}}+\frac{2 \sin ^{2} m_{1}}{3 \alpha \beta}\right\}=\frac{2 m_{1}^{2}}{1+\alpha \beta} \int_{0}^{1}\left(\Theta \theta_{1}+\beta / \alpha . \mathrm{S} s_{1}\right) r^{2} d r$,

by which $B_{1}$ is determined.

An important particular case is that where $\Theta$ is constant and accordingly $\mathbb{S}$ vanishes. Now with use of $(42)$

$$
\int_{0}^{1} \theta_{1} r^{2} d r=\frac{\sin m_{1}-m_{1} \cos n_{1}}{m_{1}^{3}}-\frac{\sin m_{1}}{3 m_{1}}=-\frac{(1+\alpha \beta) \sin m_{1}}{3 \alpha \beta m_{1}} ;
$$

so that

$$
\mathrm{B}_{1}\left\{1-\frac{\sin 2 m_{1}}{2 m_{1}}+\frac{2 \sin ^{2} m_{1}}{3 \alpha \beta}\right\}=-\frac{2 m_{1} \sin m_{1} \cdot \Theta}{3 \alpha \beta} .
$$

$\mathrm{B}_{1}, \mathrm{~B}_{2}, \ldots$ being thus known, $\theta$ and $s$ are given as functions of the time and of the space coordinates by (43), (44).

To determine the pressure in this case we have from (45)

$$
\frac{\theta+s / \alpha}{\Theta}=\frac{1+\alpha \beta}{\alpha \beta} \sum \frac{\sin ^{2} m \cdot e^{-h t}}{\sin ^{2} m+\frac{\ddot{3} \alpha \beta}{2}\left(1-\frac{\sin 2 m}{2 m}\right)}, .
$$


Mass of Air confined by Walls at Constant Temperature, 323

the summation extending to all the values of $m$ in (12). Since (for each term) the mean value of $s$ is zero, the righthand member of (51) represents also $\bar{\theta} / \Theta$, where $\bar{\theta}$ is the mean value of $\theta$.

If in (51) we suppose $\beta=0$, we fall back upon a known Fourier solution, relative to the mean temperature of a spherical solid which having been initially at uniform temperature $\Theta$ throughout is afterwards maintained at zero all over the surface. From (42) we see that in this case $\sin m$ is small and of order $\beta$. Approximately

$$
\sin m=3 \alpha \beta / m \text {; }
$$

and (51) reduces to

$$
\frac{\bar{\theta}}{\bar{\Theta}}=\frac{6}{\pi^{2}}\left(\frac{e^{-h_{1} t}}{1^{2}}+\frac{e^{-h_{2} t}}{2^{2}}+\frac{e^{-n_{3} t}}{3^{2}}+\ldots\right), \quad . .
$$

of which by a known formula the right-hand nember identifies itself with unity when $t=0$. By (9) with restoration of $a$,

$$
h=\left(1^{2}, 3^{2}, 5^{2}, \ldots . .\right) v \pi^{2} / a^{2} . . . .
$$

In the general case we may obtain from (42) an approximate value applicable when $m$ is moderately large. The first approximation is $m=i \pi, i$ denoting an integer. Successive operations give

$$
m=i \pi+\frac{3 \alpha \beta}{i \pi}-\frac{18 \alpha^{2} \beta^{2}+9 \alpha^{3} \beta^{3}}{i^{3} \pi^{3}} . . .
$$

In like manner we find approximately in (51)

$$
\frac{\sin ^{2} m(1+\alpha \beta) / \alpha \beta}{\sin ^{2} m+\frac{3 \alpha \beta}{2}\left(1-\frac{\sin 2 m}{2 m}\right)}=\frac{6(1+\alpha \beta)}{i^{2} \pi^{2}}\left\{1-\frac{15 \alpha \beta+9 \alpha^{2} \beta^{2}}{i^{2} \pi^{2}}\right\},
$$

showing that the coefficients of the terms of high order in (51) differ from the corresponding terms in (52) only by the factor $(1+a \beta)$ or $\gamma$.

In the numerical computation we take $\gamma=1 \cdot 41, \alpha \beta=\cdot 41$. The series (54) suffices for finding $m$ when $i$ is greater than 2 . The first two terms are found by trial and error with trigonometrical tables from (42). In like manner the approximate value of the left-hand member of (51) therein given suffices when $i$ is greater than 3 . The results as far as $i=12$ are recorded in the annexed table. 
324 On the Conduction of Heat in a Spherical Mass of Air.

\begin{tabular}{|c|c|c|c|c|c|}
\hline$i$. & $m / \pi$ & $\begin{array}{l}\text { Left-hund } \\
\text { member } \\
\text { of }(55) \text {. }\end{array}$ & $i$. & $m / \pi$ & $\begin{array}{l}\text { Left-hand } \\
\text { member } \\
\text { of }(55) \text {. }\end{array}$ \\
\hline $1, \ldots \ldots \ldots$ & 1.0994 & $\cdot 4942$ & †..... & $7 \cdot 0177$ & .0175 \\
\hline $2 \ldots \ldots \ldots$ & 20581 & $\cdot 1799$ & $8 \ldots \ldots$ & $8 \cdot 0156$ & .0134 \\
\hline 3......... & $3 \cdot 0401$ & 0871 & $9 \ldots \ldots$ & $9 \cdot 0138$ & .0106 \\
\hline $4 \ldots \ldots \ldots$ & $4 \cdot 0305$ & $\cdot 0510$ & $10 \ldots \ldots$ & 10.0125 & .0086 \\
\hline $5 \ldots \ldots \ldots$ & $5 \cdot 0246$ & $\cdot 0232$ & $11 \ldots \ldots$ & 11.0113 & $\cdot n 071$ \\
\hline $6 \ldots \ldots \ldots$ & 6.0206 & .0233 & $12 \ldots \ldots$ & 120104 & .0060 \\
\hline
\end{tabular}

Thus the solution (51) of our problem is represented by

$$
\bar{\theta} / \Theta=\cdot 4942 e^{-(1 \cdot 0994)^{2} t^{\prime}}+\cdot 1799 e^{-(2 \cdot 0581)^{2} t^{\prime}}+\ldots .
$$

where by (9), with omission of $q$ and restoration of $a$,

$$
t^{\prime} / t=\pi^{2} v / \gamma a^{2} . . . . . . .
$$

The numbers entered in the third column of the above table would add up to unity if continued far enough. The verification is best made by a comparison with the simpler series (52). If with $t$ zero we call this series $\Sigma^{\prime}$ and the present series $\Sigma$, both $\Sigma$ and $\Sigma^{\prime}$ have unity for their sum, and accordingly $\gamma^{\prime}-\Sigma=\gamma-1$, or

$$
\frac{6 \gamma}{\pi^{2}}\left(\frac{1}{1^{2}}+\frac{1}{2^{2}}+\frac{1}{3^{2}}+\ldots\right)-\Sigma=\gamma-1=\cdot 41 \text {. }
$$

Here $6 \gamma / \pi^{2}=\cdot 8573$, and the difference between this and the first term of $\Sigma, i . e \cdot \cdot 4942$, is $\cdot 3631$. The differences of the

\begin{tabular}{|c|c|c|c|}
\hline$t^{\prime}$ & (5 i). & $t^{\prime}$ & (56). \\
\hline $.00 \ldots \ldots \ldots$ & $1-0000$ & $90 \ldots .$. & .2538 \\
\hline $.05 \ldots \ldots \ldots$ & $\cdot 7037$ & $\cdot 70 \ldots \ldots \ldots$ & .2215 \\
\hline$\cdot 10 \ldots \ldots \ldots$ & $\cdot 6037$ & $80 \ldots \ldots \ldots$ & $\cdot 1940$ \\
\hline$\cdot 20 \ldots \ldots \ldots$ & $\cdot 4811$ & $90 \ldots \ldots \ldots$ & $\cdot 1705$ \\
\hline$\cdot 30 \ldots \ldots \ldots$ & $\cdot 4002$ & $1.00 \ldots \ldots \ldots$ & $\cdot 1502$ \\
\hline $40 \ldots \ldots \ldots$ & 3401 & $150 \ldots \ldots$ & .0809 \\
\hline $50 \ldots \ldots \ldots$ & $\cdot 2926$ & $2 \cdot 00 \ldots \ldots \ldots$ & .0441 \\
\hline
\end{tabular}
second, third, \&c. terms are $\cdot 0344, \cdot 0082, \cdot 0026, \cdot 0011, \cdot 0005$, $\cdot 0000, \& c .$, making a total of $\cdot 4099$.

We are now in a position to compute the right-hand 
member of (56) as a function of $t^{\prime}$. The annexed table contains sufficient to give an idea of the course of the function. It is plotted in the figure. The second entry $\left(t^{\prime}=\cdot 05\right)$ requires

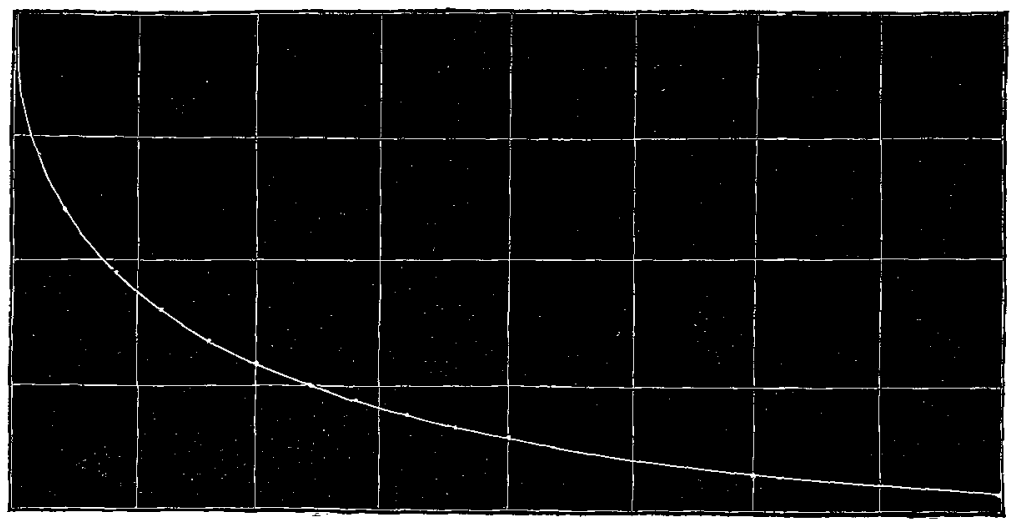

the iuclusion of 9 terms of the series. A fter $t^{\prime}=\cdot 7$ two terms suffice; and after $t^{\prime}=2 \cdot 0$ the first term represents the series to four places of decimals.

By interpolation we find that the series attains the value 5 when

$$
\begin{aligned}
& t^{\prime}=\cdot 184 . \quad \text {. . . . . }(58)
\end{aligned}
$$

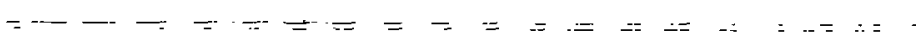

XXVI. Notices respecting New Books.

An Elementary Course in the Intesral Calculus. By Dr. D. A. Mureay, Comell University. Longmans, 1898. Pिp. $\mathrm{x}+288$.

$D^{R . M L R R A Y}$ states his object to be to present " the subjertmatter, which is of an elementary churacter, in a simple manner." This he bas succeeded in doing, and the work is wellarranged and the explanations given are exceedingly clear. In Chapter I. he trcats Integration as a process of summation, and in Chapter II. as the inverse of differentiation. 'The author's olject herein is to give the student a clear idea of what the Integral Calcul s is, and of the uses to which it may be applied. The first ten chapters are devoted to a treatuent of the mat ters handled in such works as Williamson's, Edwards's, and other woll-known treatises. Chapter XI. treats of approximate integration, and the application of the Calculus to the measurement of areas. Here we bave clear statements and proofs of the trapezoidal rule, Simpsou's onethird rule, and Durand's rule. To this latter gentleman the atuthor is indebted for valuable suggestious of use to engineering students. Prof. Durand has also put at Dr. Murray's disposal his article on "Integral Curves" (in the "Sibley Journal of 